\title{
Design and Fabrication of Robotic Gripper
}

\author{
Hamidreza Heidari*, Milad Jafary Pouria, Shahriar Sharifi
}

\begin{abstract}
This paper presents a new method for the improvement and design of the kinematic structure of a robotic hand for successful grasping of objects in unstructured environments. The main goal is to improve kinematic structure of gripper to increase the grasping capability of large objects and makes a successful grasp of various objects in unstructured environments. Our model contains two fingers with combining a kinematic model of thumb-index. Moreover, some experimental tests are performed to examine the effectiveness of the hand-made in real, unstructured tasks. Our findings show that the presented method provides an accommodative kinematic model which makes the better grasping by fingers geometries for a human-like robotic hand.
\end{abstract}

Keywords - Mechanical compliance, robotic hand, unstructured environments.

\section{INTRODUCTION}

Robotic manipulators for space applications are now a wellestablished technology in space applications, as they are routinely used for handling and assembling large space modules and for reducing human extravehicular activities at the international space station. The grasp of elements or objects in unstructured environments is one of the important challenges in robotics device (Hubbard, 2005). Recently, there has been an appropriate innovation in field of adaptive grasping for design hands by utilized passive mechanical compliance and under-actuated in the finger and hand structure.

A number of researchers in robotics have used the concept of employing mechanical compliance and passive adaptability to grasp a variety of objects successfully. Dollor and Howe (2005) presented the design optimization studies in which the kinematic structure, compliance configuration, and joint coupling are applied. These results in a successful grasp while contact forces is low [1]. Kragten and Herder (2010) investigated the design of an under-actuated compliant finger with a monolithic structure as compliance is distributed largely [2]. Toglia et al. (2011) described a class of maneuvers that, given the same target to be grasped, are characterized by different mission objectives [3]. Ma et al. (2013) presented the design of an open-source, low-cost, single actuator with under-actuated hand. They established the design of an adaptive, four-finger hand utilizing simple 3D printed components, compliant flexure joints, and readily obtainable off-the-shelf parts [4].

Assistant Professor, Department of Mechanical Engineering, Malayer University, Malayer, Iran
Carbone and Ceccarelli (2008) investigated the design of LARM Hand to develop a low-cost easy-operation robotic hand [5]. The normal and tangential stiffnesses of soft materials have been experimentally investigated by Biagiotti1 et al. (2005) in order to demonstrate their suitability with the development of compliant pads for robotic hands [6]. Deimel and Brock (2016) constructed a highly compliant, underactuated, robust and dexterous anthropomorphic robotic hand based on soft robotics technology [7]. Boohene et al. (2015) expanded a new design of the original gripper to conform to irregular surface variations, providing improvements in anchoring performance and creating a dexterous robotic hand [8].

Feix et al. (2015) presented a kinematic model of thumbindex precision grip and manipulative movement based on bony hand morphology in a broad sample of extant primates and fossil hominins [9]. Backus et al. (2015) studied a grasping behavior of a simplified bird foot in response to the downward and upward forces imparted by carrying and perching tasks, respectively. They explicitly, compared the performance of various foot geometries in performing these tasks when only actuated by distally inserted flexors, for both distally and proximally inserted flexors [10].

In this paper, according to the structure of human fingers, hand kinematic and structure including a three-link similar to the human index finger and a two-link similar to the thumb has been designed and fabricated. To analysis our fabricated hand, equations of each finger configuration were extracted in the section 2. Then, effective parameters such as spring rest link angle, joint stiffness value and object radius were optimized and extracted. In addition, some experiments about the performance of this compliant under-actuated two-fingered hand were performed for grasping a number of variety target objects. Finally, this study demonstrate that the kinematic structure of the human hand the significantly enhances the performance of the gripper for a successful grasp of objects.

\section{GRASPING KINEMATIC AND DYNAMIC EQUATIONS}

In this study, a simple two-finger gripper was proposed for grasping the objects. As shown in the Fig. 1, this griper model includes a two-link finger with two revolute degrees of freedom similar to the thumb and a three-link finger with three revolute degrees of freedom similar to the index. In our model, it is assumed that links are rigid lines between joints and each joint of the gripper includes a passive linear spring in series with an actuator. In order to have a successful grasping, at least two-point contact with the object is needed for the threelink finger. 


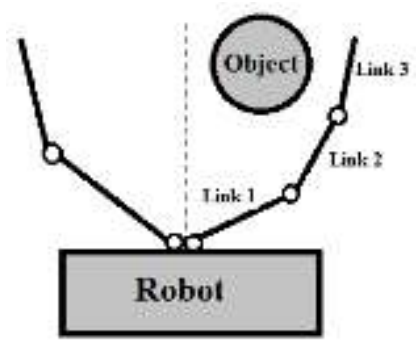

Fig. 1 Schematic view of two fingers

One of the crucial challenges for grasping system is analyzing the deflection of the grasper finger due to contact with the object as the robot advances. Indeed, three different modes of object contact with a finger are possible:

\section{A. Mode 1: Initial Contact at the tip of distal link:}

In the first case, object contacts with the tip of distal-link (link 3). In the presence of friction, the tip will stick and maybe roll until static friction is overcome. As the robot moves forward, it begins to slide and possibly transitions to contact along the length of link 2 (the second case described below). A successful grasp will not often be achieved in this case.

\section{B. Mode 2: Initial Contact on the distal link:}

In the second case, initial contact occurs on distal-link (Fig. 2). In this condition, the robot must continue moving forward to roll and slide the object along the length of link 3 until the object is successfully grasped. However, the possibility of achieving two-point contact on the grasper of three-link finger is subtle. An enveloping grasp can be achieved is, therefore, the criterion by which a successful grasp configuration is judged in this analysis.

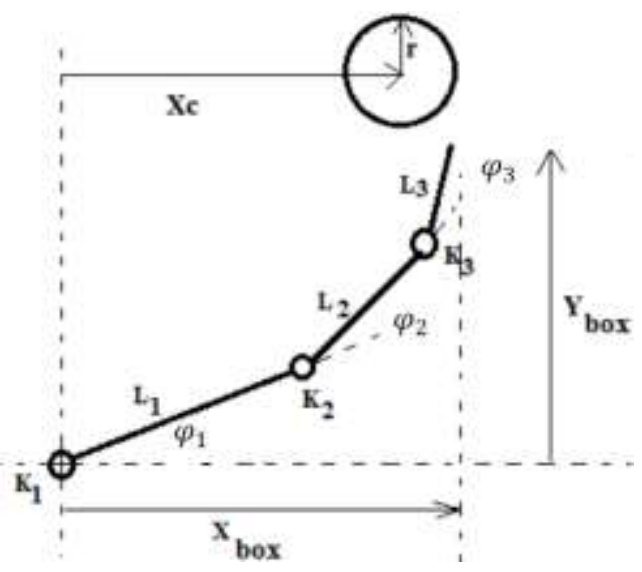

Fig. 2 The manipulator before contact with the object

The inverse kinematics of the mechanism along with a torque balance for each joint must be solved. The final equations describe the geometry of the grasper and object. Initial contact on the distal link will define the coordinates of the initial contact point. This coordinate is a point where the first contact of the object with the distal link has established.

$$
\begin{gathered}
x_{0}=r \sin \left(\varphi_{1}+\varphi_{2}+\varphi_{3}\right)+x_{c} \\
y_{0}=l_{1} \sin \varphi_{1}+l_{2} \sin \varphi_{2}+ \\
\left(x_{0}-l_{1} \cos \varphi_{1}-l_{2} \cos \varphi_{2}\right) \tan \left(\varphi_{1}+\varphi_{2}+\varphi_{3}\right)
\end{gathered}
$$

After initial contact occurred and robot moves forward for sliding and rolling the object on link 3, contact point coordinates have been changed and geometry of the object and finger for this coordinate are as follows:

$$
\begin{gathered}
x_{3}=r \sin \left(\varphi_{1}+\varphi_{2}+\varphi_{3}+\theta_{1}+\theta_{2}+\theta_{3}\right)+x_{c} \\
y_{3}=y_{0}-y+r \cos \left(\varphi_{1}+\varphi_{2}+\varphi_{3}\right) \\
-r \cos \left(\varphi_{1}+\varphi_{2}+\varphi_{3}+\theta_{1}+\theta_{2}+\theta_{3}\right)
\end{gathered}
$$

After object slides on link 3, the equations of the contact point coordinates can be extracted by using kinematic as follows:

$$
\begin{gathered}
x_{3}=l_{1} \cos \left(\varphi_{1}+\theta_{1}\right)+l_{2} \cos (\beta)+a_{3} \cos \left(\alpha^{\prime}\right) \\
y_{3}=l_{1} \sin \left(\varphi_{1}+\theta_{1}\right)+l_{2} \sin (\beta)+a_{3} \sin \left(\alpha^{\prime}\right)
\end{gathered}
$$

According to final torque balance for each joint, following equations will be obtained:

$$
\begin{aligned}
& \quad F_{n} a_{3}=-k_{3} \theta_{3} \\
& -k_{2} \theta_{2} \\
& =F_{n} \sqrt{\left(x_{3}-l_{1} \cos \left(\varphi_{1}+\theta_{1}\right)\right)^{2}+\left(y_{3}-l_{1} \sin \left(\varphi_{1}+\theta_{1}\right)\right)^{2}} \\
& \times\left[\sin (\alpha)+\tan ^{-1}\left(\frac{x_{3}}{y_{3}}\right)+\mu_{k} \cos \left(\pi-\alpha-\tan ^{-1}\left(\frac{x_{3}}{y_{3}}\right)\right]\right. \\
& -k_{1} \theta_{1}=F_{N} \sqrt{x_{3}^{2}+y_{3}^{2}}\left[\operatorname { s i n } \left(\alpha^{\prime}+\tan ^{-1}\left(\frac{x_{3}}{y_{3}}\right)+\mu_{k} \cos (\pi\right.\right. \\
& \left.-\alpha^{\prime}-\tan ^{-1}\left(\frac{x_{3}}{y_{3}}\right)\right] \\
& \beta=\varphi_{1}+\varphi_{2}+\theta_{1}+\theta_{2} \\
& \alpha=\varphi_{2}+\varphi_{3}+\theta_{2}+\theta_{3} \\
& \alpha^{\prime}=\varphi_{1}+\varphi_{2}+\varphi_{3}+\theta_{1}+\theta_{2}+\theta_{3}
\end{aligned}
$$

Also, the following constraints are considered as restrictions on the movements. In fact, these conditions confirm that the links are able to successful grasp and do not deflect past the line horizontal from the base joint.

$$
\begin{aligned}
& \varphi_{1}+\theta_{1} \geq 0 \\
& \varphi_{2}+\theta_{2} \geq 0 \\
& \varphi_{3}+\theta_{3} \geq 0
\end{aligned}
$$

\section{C.Mode 3: Initial Contact on the central or internal link:}

The contact on link 1 or link 2 is the simplest and best mode for the successful grasp. In this case, joint 2 and joint 3 immediately actuated to achieve three-point contact and successfully grasp the object. For this contact mode, the equations of two-link finger initial point coordinate for the contact with surface of the central-link has achieved on Ref. 
[1]. As the robot advances, object slides and rolls on the link 2. The trice coordinates of contact point between the object and central-link will be result.

$$
\begin{gathered}
x_{0}=r \sin \left(\varphi_{1}+\varphi_{2}\right)+x_{c} \\
y_{0}=l \sin \varphi_{1}+\left(x_{0}-l \cos \varphi_{1}\right) \tan \left(\varphi_{1}+\varphi_{2}\right) \\
x_{2}=r \sin (\beta)+x_{c} \\
y_{2}=y_{0}-y+r \cos \left(\varphi_{1}+\theta_{1}\right)-r \cos (\beta) \\
x_{2}=l \cos \left(\varphi_{1}+\theta_{1}\right)+a_{2} \cos (\beta) \\
y_{2}=l \sin \left(\varphi_{1}+\theta_{1}\right)+a_{2} \sin (\beta)
\end{gathered}
$$

And finally torque balance for each joint following equation will result:

$$
\begin{gathered}
-k_{2} \theta_{2}=f_{N} a_{2} \\
\frac{-k_{1} \theta_{1}}{f_{N} \sqrt{x_{2}+y_{2}}}=\sin \left[\beta+\tan ^{-1}\left(\frac{x_{2}}{y_{2}}\right)+\mu_{k} \cos [\pi-\beta\right. \\
\left.-\tan ^{-1}\left(\frac{x_{2}}{y_{2}}\right)\right]
\end{gathered}
$$

\section{OPTIMIZATION PARAMETERS}

In order to design an appropriate model for the geometry of the gripper, variable parameters such as object radius $(r)$, spring rest link angles $(\varphi)$ and the Joint stiffness values $(k)$ are defined in the hand geometric structure. Due to these variables, the objective function of this paper is to determine the distance from the center of the circle to the centerline of the grasper $\left(x_{c}\right)$.

For the kinematic structure analysis of three-link finger, the optimal rest link angles and also the ratio of Joint stiffness were used as constant values $\left(\varphi_{1}=20^{\circ}\right.$ and $\left.k_{\alpha}=0.5\right)$ based on Pollard and Gilbert (2002). Then, rest of the parameters $\left(\varphi_{2}, \varphi_{3}\right.$ and $\left.k_{\beta}\right)$ associated with three-link finger was evaluated and the simulation was implemented in MATLAB according to the variation of these parameters. Then, optimize value of each parameters was obtained. Since $\varphi_{1}$ is fixed, variations in angles of $\varphi_{2}$ and $\varphi_{3}$ decline 20 degree from maximum amount of 90 degree and $\varphi_{2}$ and $\varphi_{3}$ were chosen to be the varied geometric parameters. These angles were varied from 0 to 70 degrees at 5-degree increments (more than this amount cause unsuccessful grasping). Also, the static and kinetic friction coefficients were set equal to reduce the dimension of the space parameter. In the literature, the ratio of joints stiffness should be less than 1 for optimal conditions of finger structure. This means that internal joints have less joint stiffness from distant joint. Hence, amount of $k_{\beta}$ was considered at values $0.3,0.6$ and 1 .

To compare the results of the three-link finger with two-link finger, it is assumed that the amounts of opening both fingers toward the center line are equal. For the three-link finger which has similar size with the human index finger, the finger opening of the central line is equal to $70.15 \mathrm{~mm}$. To keep this opening for the two-link finger, the length of each link should be $56.2 \mathrm{~mm}$. For address this need, the amount of possible change for the variable parameter $r / l$ in order to have a successful grasping is monitored to obtain a successful grasping. The relative amounts of radiuses proposed by Dollar and Howe (2005) are 0.1, 0.5, and 0.9.

After solving kinematic and dynamic equations simultaneously, the values of $\theta_{1}$ and $\theta_{2}$ are obtained as a function of $\mathrm{y}$. The performance of each mechanism configuration was evaluated for the range of design variables $\left(\varphi_{2}, \varphi_{3}, r / l\right.$ and $\left.k_{\beta}\right)$ and object location, $x_{c} / l$, . It is found that 0.01 incremented from the center toward the outside of the grasping range is obtained due to the geometric constraints (i.e., $\varphi_{1}+\theta_{1}=0$ ). Moreover, the maximum amounts $x_{c} / l$ for a successful grasp were extracted from MATLAB for each configuration. This value represents the successful grasp range. Finally, the largest contact force applied to the object during the grasping process was also recorded for each object location, $x_{c}$. This information was used to calculate the average maximum contact force over the grasp range for each tested grasper configuration.

The comparison of the results shows that increasing the stiffness $\left(\mathrm{k}_{\beta}\right)$ does not affect the maximum value of the successful grasp range, $\left(x_{c}\right)_{\max }$. In particular, a broader range of values for $\varphi_{2}$ and $\varphi_{3}$ produce the maximum grasp range if the distal joint is stiffer than the middle joint $\left(k_{2}<k_{3}\right)$.

Eventually, the optimum configuration space slightly changes with object radius and becomes smaller and tends to increase $\varphi_{3}$ for raising object radius. Thus, the value of $\left(x_{c}\right)_{\max }$ decreases when $\varphi_{2}$ or $\varphi_{3}$ increases. As a result, the grasp range is particularly sensitive to variations in the distal joint of rest angle, $\varphi_{3}$, while variations in $\varphi_{2}$ are not significant for small values of the stiffness $k_{\alpha}$.

\section{HAND FABRICATION AND ACTUATION}

The goal in the fabrication of this hand was to obtain the simplest possible hand for robust grasping and manipulation of various objects in different locations. In this approach, according to the results of this study and previous conducted optimization works, a basic two-fingered hand structure was fabricated as shown in Fig. 3. Since two is the smallest number of fingers that can be used for grasping objects, this model is chosen. To provide a 3D model of the hand, mechanical design software was used. The proposed hand is fabricated by using 3D printing which is an appropriate method for rapid-prototyping fabrication with low-cost and high accuracy.

\section{A. Finger fabrication}

In this section, a brief description on fabrication process of the hand fingers including a two-link finger similar to the thumb and a three-link finger similar to the index is presented. The robot index finger is consisted of three phalanges (proximal, middle and distal) and three Degrees of Freedom (DoF) while the robot thumb is made up of two phalanges (proximal and distal) and two DoFs. For robot index, finger is long for each phalange like the human index finger and the 
length of these phalanges (measured from the center of the joint flexures) are 47, 25.7 and $22.4 \mathrm{~mm}$, respectively. Also, each links of the two-link finger are $42 \mathrm{~mm}$ long. In terms of hand design, tip of two fingers is inserted in a state of complete disruption along a horizontal line and being equal with the opening of robotic hand similar to the human hand.

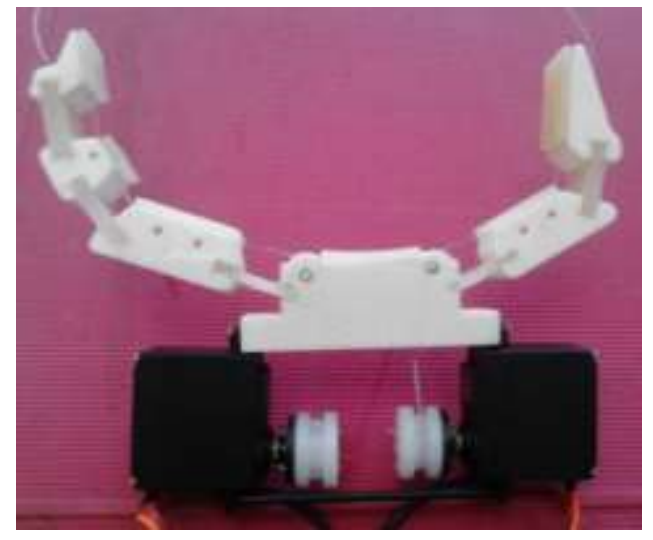

Fig. 3 A compliant, under-actuated robotic hand, capable of successful grasping

The concave side of each link contains a soft finger pad to maximize the friction and contact area, and thereby increase the grasp stability (Dollar and Howe 2005). Links are connected with elastomeric joint flexures and the polyurethane used for these joints (described in detail in Dollar and Howe 2010). They are designed to be compliant in the plane of the finger motion and stiffer out of the plane. In this study, each links of the finger was fabricated by PLA (Poly Lactic Acid) which is light-weight, robust and low-cost. The polyurethane is also used for the joints to demonstrate significant viscoelastic behavior by providing both compliance and passive damping to the hand. Therefore, our hand is robust by use of compliance and polymeric construction.

In order to provide the human-like robotic hand, the appropriateness of the physical size is crucial. Thus, in this study the tip of fingers is aligned with a horizontal line with $170 \mathrm{~mm}$ length. To accomplish this, the fingers are staggered in the out-of-plane direction on the palm; it is also made of PLA, to allow them to completely close without interfering with one another.

\section{B. Actuation}

The actuators were chosen to minimize the cost and control complexity with an appropriate authority. Each of the two fingers is driven by using a single tendon through running the length of the finger. For each finger, the extension is mechanically implemented in a passive fashion through the use of appropriate elastomeric materials. Meanwhile, the flexion is implemented with cables (made of fishing line) so that tendon can handle about $270 \mathrm{~N}$. Therefore, two actuators drive the two fingers (five joints) of the hand. The actuators are servo MG 995 which has a maximum torque output of 1.21.4 Nm. With a drive pulley diameter of $7 \mathrm{~mm}$, the servo $\mathrm{MG}$ 995 can apply a maximum tendon force of $200 \mathrm{~N}$ for each finger in this design. Hence, fingers velocity is between 1- 2 $\mathrm{cm} / \mathrm{s}$ (centimeter per second).

\section{V.EXPERIMENTAL EVALUATION}

This section presents an overall evaluation of the approach followed in this study and discusses the main insights that were gained into grasping. The performance of this hand is to grasp a large range of objects with different sizes, shapes and weights in different conditions. Fig. 4 clearly shows how the hand grasps various objects with different geometries. The evaluation is substantiated by appropriate performance of this hand with the capability to grasp a range of objects when inherent uncertainty associated with unstructured environments makes establishing a successful grasp difficult. In the following section, two experiments are described in order to quantify the performance of the mechanical hand in the grasp range and contact force experiment. In the first, a comparison between each fingers have performed to obtain maximum distance from the center of the circle to the centerline of the grasper. It defined how these fingers act on grasping large objects. In the second stage, the test is done to investigate the forces of fingers during contact with a target object and compares these forces applied by each finger.

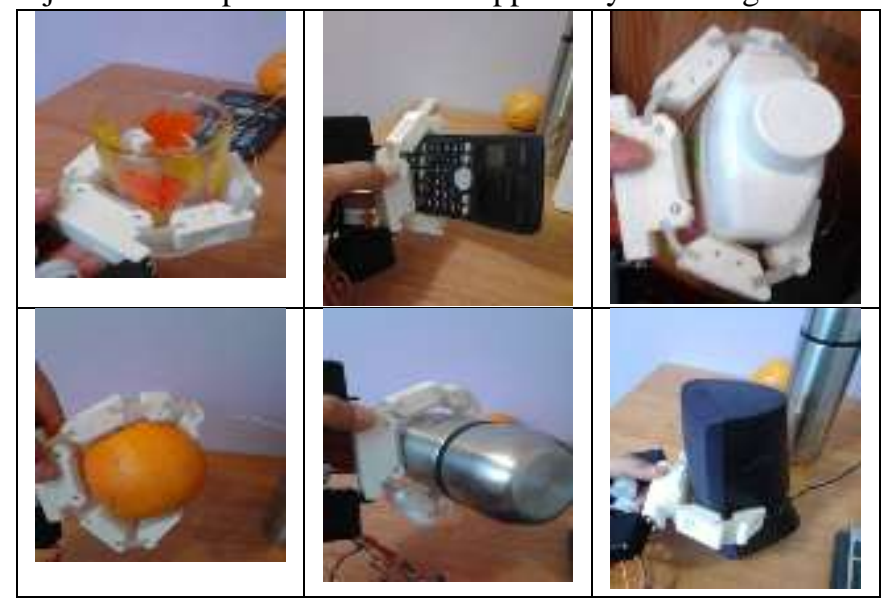

Fig. 4 The set of passive adaptive grasps that gripper was designed to perform is shown above, including cylindrical, spherical and opposed pinch grasps, as well as cylindrical and spherical power grasps

\section{CONCLUSIONS}

In this study, a new method is proposed to design and improve of the kinematic structure of a robotic hand for successful grasping in unstructured environments. The results demonstrate the impact of improvement kinematic on grasp parameters such as spring rest link angles, object diameter and relative link lengths. They also done examined to evaluate the relative performance of gripper when robotic hand is in unstructured environments. According to the results, it is found that a two fingers hand is sufficient device for most grasping tasks, and the hand is able to grasp various objects with unknown properties. Comparison defined the maximum normalized distance of the object from the centerline $(\max (x c / 1))$ of gripper for two and three link finger with symmetric two link fingers gripper (Dollar and Howe, 2005). 
The results also represent that the range of allowable positioning errors is improved about $30 \%$ in the object size of the two-link finger. In addition, the range of grasping large objects for symmetrical two-fingered gripper is about 0.46 (Dollar and Howe, 2005) and this amount have been improved to 0.6 for our gripper. Moreover, the results of the simulation confirmed that the use of a three-link finger decreases the contact forces approximately $10 \%$ during the initial stages of the grasping of large objects between the object and finger. According to obtained results, the use of the three-link finger for the fabrication of a two-fingered gripper will improve the contact forces and grasping situation.

\section{REFERENCES}

[1] Dollar AM and Howe RD (2005) Towards grasping in unstructured environments: grasper compliance and configuration optimization. Advanced Robotics 19(5): 523-543.

[2] Kragten GA and Herder JL (2010) A platform for grasp performance assessment in compliant or under-actuated hands. Journal of Mechanical Design 132(2): 1-6.

[3] Toglia C, Sabatini M, Gasbarri P and Palmerini GB (2011) Optimal target grasping of a flexible space manipulator for a class of objectives. Acta Astronautica 68(1): 1031-1041.

[4] Ma RR, Odhner LU and Dollar AM (2013) A modular, open-source 3D printed underactuated hand. In: IEEE International Conference on Robotics and Automation (ICRA), Karlsruhe, Germany, 6-10 May, pp. 12-14.

[5] Carbone G and Ceccarelli M (2008) Design of LARM hand: problems and solutions. In: International Conference on Automation, Quality and Testing, Robotics, Cluj-Napoca, Romania, 22-25 May, pp. 298-303.

[6] Biagiotti L, Tiezzi P, Vassura G and Melchiorri C (2005) Modelling and controlling the compliance of a robotic hand with soft finger-pads. Multi-point Interaction with Real and Virtual Objects 18(1): 55-73.

[7] Deimel R and Brock O (2016) A novel type of compliant, underactuated robotic hand for dexterous grasping. The International Journal of Robotics Research 35(3): 161-185.

[8] Boohene ANK, Smith DN, Trieu T and Stengel R (2015) Prototype for an asteroid exploratory robot using multi-phalanx microspine grippers. In: AIAA SPACE Conference and Exposition, California, USA, 1-2 September, pp. 4585:1-21.

[9] Feix T, Kivell TL, Pouydebat E and Dollar AM (2015) Estimating thumb-index finger precision grip and manipulation potential in extant and fossil primates. Journal of the Royal Society Interface 12(106): 112.

[10] Backus SB, Sustaita D, Odhner LU and Dollar AM (2015) Mechanical analysis of avian feet: multi-articular muscles in grasping and perching. Journal of the Royal Society Open Science 2(2): 1-17. 